\title{
New loss of mains detection algorithm for embedded generation using rate of change of voltage and changes in power factors.
}

\section{Salman, S. K., King, D.J. and Weller, G.}

This is a paper presented at the $7^{\text {th }}$ International Conference on developments in power systems protection (DPSP 2001), Amsterdam, Netherlands, 9-12 April 2001

This version is a postprint of a paper submitted to and accepted for publication in Proceedings of the 7th International conference on developments in power system protection (DPSP 2001) and is subject to Institution of Engineering and Technology Copyright. The copy of record is available at IET Digital Library, doi: 10.1049/cp:20010105 


\title{
NEW LOSS OF MAINS DETECTION ALGORITHM FOR EMBEDDED GENERATION USING RATE OF CHANGE OF VOLTAGE AND CHANGES IN POWER FACTORS
}

\author{
S. K. Salman ${ }^{(1)}$, D. J. King ${ }^{(1)}$ and G. Weller ${ }^{(2)}$ \\ (1) The Robert Gordon University, UK, (2) ALSTOM T\&D, UK
}

\begin{abstract}
The work reported in this paper proposes a new method for detecting Loss of Mains (LOM), which considered one of the basic protection system that should be provided for an Embedded Generator (EG). The proposed LOM protection particularly consider the case when the EG is "islanded" with part of the utility load which has a capacity close to that of the generator. It also has the capability to deal with the difficult situation related to differentiate between LOM and the loss of parallel feeder at the interfacing circuit between EG and the utility. The proposed method is based on using the Rate of Change of Voltage $(d V / d t)$ and the change in Power Factor $(\Delta p f)$ measured at the interfacing point between the EG and the utility network.
\end{abstract}

\section{INTRODUCTION}

The protection problems caused as a result of integration of embedded generator(s) (EGs) into utilities' network at distribution level have been well documented, especially the need to immediately detect LOM and disconnect the EG $(1,2)$. The particular condition which needs to be avoided is when an EG becomes islanded with part of the public network load. If the load is closely matched to the EG capacity, then the EG may continue to operate in a power island. This is highly undesirable due to safety as well as technical considerations (3-5). For example the quality of power supply to the customers can not be guaranteed in the islanded system.

In recent years much research has been done in an effort to provide suitable solution for EG's protection against the adverse effects caused by $\operatorname{LOM}(2,6-10)$. However, these methods are either in the development stage, or are in use but still prone to maloperation, particularly the case when the islanded load matches the EG capacity [10-12].

The proposed LOM reported in this paper is addressing among other conditions the most difficult situation with regard to LOM detection, whereby the magnitude of the islanded load is comparable to that of EG in the island.

\section{SYSTEM SIMULATION}

Figure 1 shows the single line diagram of the network used in this investigation. It basically consists of EG connected to a Grid with an assummed fault level of
3436 MVA. The EG comprises of ten 2.5 MVA, $11 \mathrm{kV}$ synchronous generators, which are modelled by a single 25 MVA generator. It is simulated using EMTP. The Grid is modelled as a fixed voltage source at $0^{\circ}$ phase angle, thus acting as a slack bus during load flow calculations, and as a phase angle reference for the rest of the network. The grid impedance between buses 1 and 2 is based on an $X / R$ ratio that could be changed to suit each individual simulation. This system allows the representation of (i) LOM and islanding condition with Load 2 by switching off $\mathrm{O} / \mathrm{H}$ lines 1 and 3, (ii) fault condition by the closure of the switch marked "FAULT" and (iii) other disturbances due to the connection and/or disconnection of generation and load represented by generator GEN 1 and LOAD 1 respectively.

In order to differentiate between LOM and other disturbance conditions, six cases were considered. Case 1 is LOM, where overhead line 3 is switched off and a three-phase fault mid-way along overhead line 1 is simulated by closing the switch "FAULT" $0.3 \mathrm{sec}$. into the simulation. The circuit breakers on overhead line 1 are then opened after $0.35 \mathrm{sec}$. leaving load 2 "islanded" with the EG.

Case 2 is the same as case 1 except this time a disturbance is assumed instead of fault condition by the closure of the switch for GEN 1 (120 MVA) after 0.35 sec. Case 3 is similar to case 2, except that the switch is closed from the start of the simulation, and is opened after $0.35 \mathrm{sec}$. This time GEN 1 is 200 MVA. Cases 4 and 5 are similar to cases 2 and 3 except that this time the switch to LOAD 1 is operated. For case 4 LOAD 1 is $630 \mathrm{MVA}$, for case 5 it is $80 \mathrm{MVA}$.

Case 6 simulating the case of loss of parallel feeder (LOPF). In this case it is assumed that the two overhead

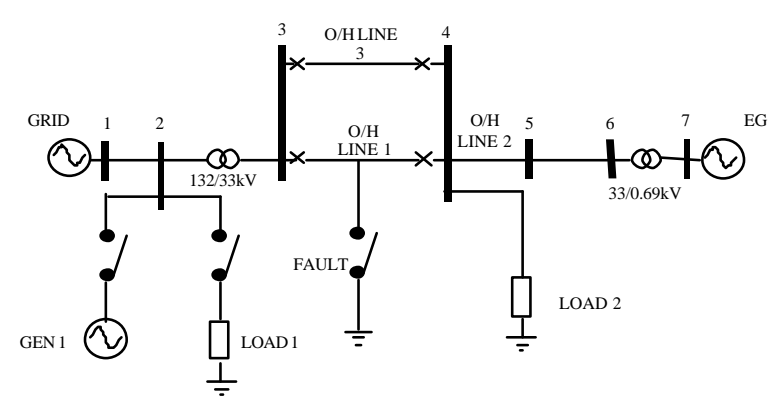

Figure 1 Single line diagram of the simulation network 
lines 1 and 3 are initially connected, but due to a fault condition on line 1 (similar to case 1 ), the line is tripped.

These six cases were simulated for nine different system permutations, by changing the grid $X / R$ ratio and the value of LOAD 2 as detailed below:

System 1 . Grid $X / R=20 / 1, \mathrm{EG} \cong \mathrm{LOAD} 2 \cong 18 \mathrm{MVA}$

System 2 . Grid $X / R=20 / 1, \mathrm{EG} \cong 18 \mathrm{MVA}, \mathrm{LOAD} 2 \cong$ 35 MVA

System 3. Grid $X / R=20 / 1, \mathrm{EG} \cong 18 \mathrm{MVA}, \mathrm{LOAD} 2 \cong$ 2.5 MVA

System 4. Grid $X / R=2 / 1, \mathrm{EG} \cong \mathrm{LOAD} 2 \cong 18 \mathrm{MVA}$

System 5 . Grid $X / R=2 / 1, \mathrm{EG} \cong 18 \mathrm{MVA}, \mathrm{LOAD} 2 \cong 35$ MVA

System 6. Grid $X / R=2 / 1, \mathrm{EG} \cong 18 \mathrm{MVA}, \mathrm{LOAD} 2 \cong$ 2.5 MVA

System 7. Grid $X=Z, R=0, \mathrm{EG} \cong \mathrm{LOAD} 2 \cong 18 \mathrm{MVA}$

System 8 . Grid $X=Z, R=0, \mathrm{EG} \cong 18 \mathrm{MVA}, \mathrm{LOAD} 2 \cong$ 35 MVA

System 9. Grid $X=Z, R=0, \mathrm{EG} \cong 18 \mathrm{MVA}, \mathrm{LOAD} 2 \cong$ 2.5 MVA

This gave a total of 54 cases indicated by a two-digit number, the first digit being the case number, and the second digit being the system number. Thus case 1.1 would be a LOM case with grid $X / R$ ratio of $20 / 1$ and the EG and load 2 closely matched at 18 MVA. While case 6.9 refers to a LOPF case where $X=Z$ and $R=0$ and load $2=2.5$ MVA (i.e. much smaller than the EG capacity).

\section{SIMULATION RESULTS}

The results obtained from each simulation were carefully considered and the most sensitive variables to system disturbances were monitored at the LOM point (bus 5) using TACS devices that are available in the EMTP. Eight different variables had been identified as the most sensitive to system disturbances. These are $d V / d t, d I / d t, d Z / d t, \Delta V, \Delta I, \Delta I L, \Delta Z$ and $\Delta p f$. The maximum value reached by these variables during the $0.05 \mathrm{sec}$. after each disturbance were measured and plotted on scatter graphs. These graphs were then examined to see if the LOM cases could be differentiated from the other disturbances. In most occurrences it was not possible, in particular the LOPF case appeared to be almost identical to LOM. However, figures 2 and 3 show the graphs for $d V / d t$ and $\Delta p f$, which tell a different story. Each graph shows the results split between the six main cases.

Figure 2 shows that for the $d V / d t$ variable a horizontal line drawn at approximately $100 \mathrm{kV} / \mathrm{sec}$. will differentiate all the LOM incidences (case 1) from all the other disturbances apart from LOPF (case 6). From figure 3 the two horizontal lines at approximately -0.05 and $-0.15 \mathrm{pu}$ indicates that for the $\Delta p f$ variable all the LOM cases lie between these two values (12).

\section{DEVELOPMENT OF A LOM DETECTOR}

It can be seen from figures 2 and 3 that $d V / d t$ can clearly distinguish between results in cases 1 and those related to cases 2 and 4, as all case 1 results are positive while

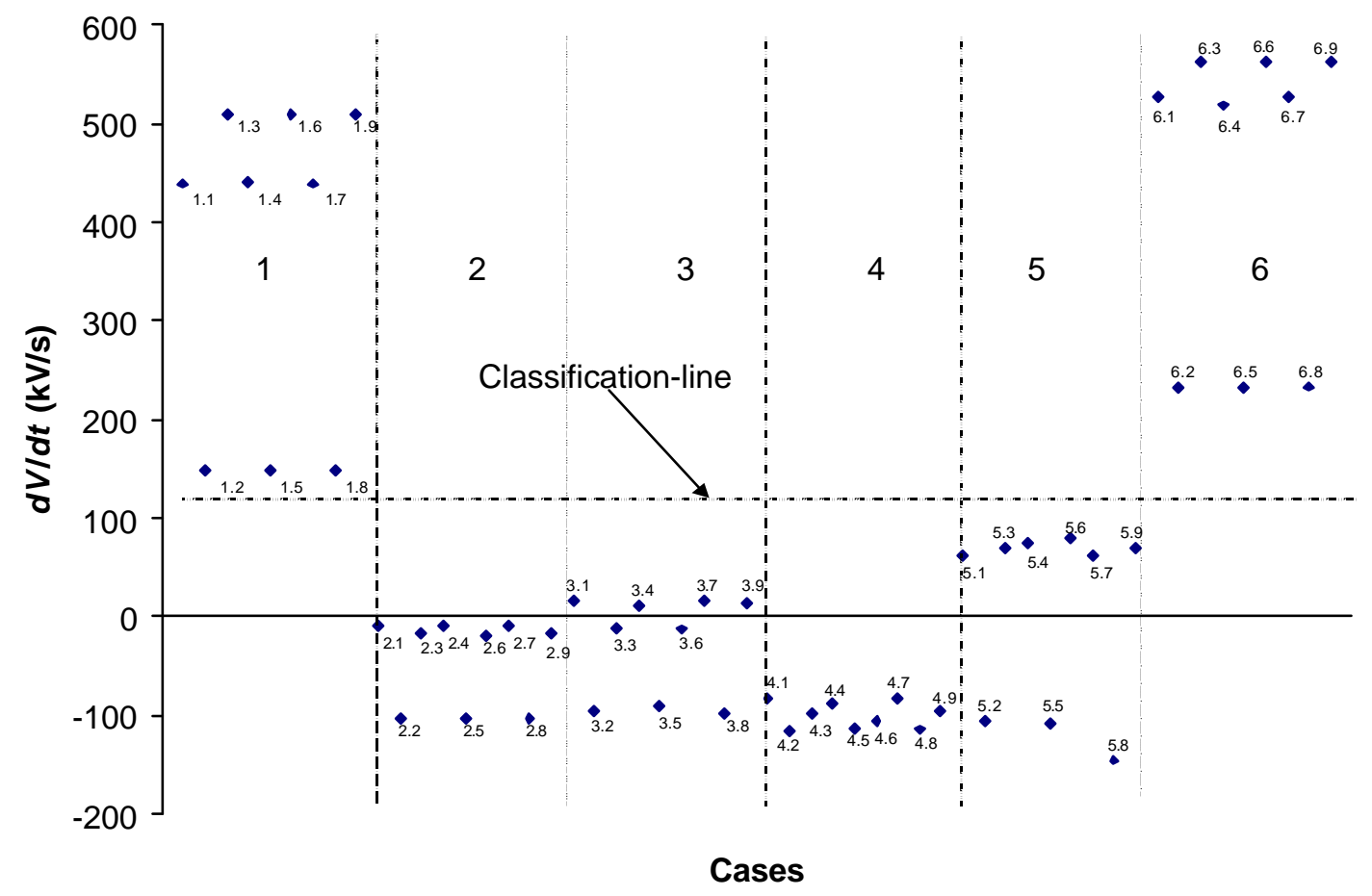

Figure 2 Plot of MaximumdV/dt Values. 


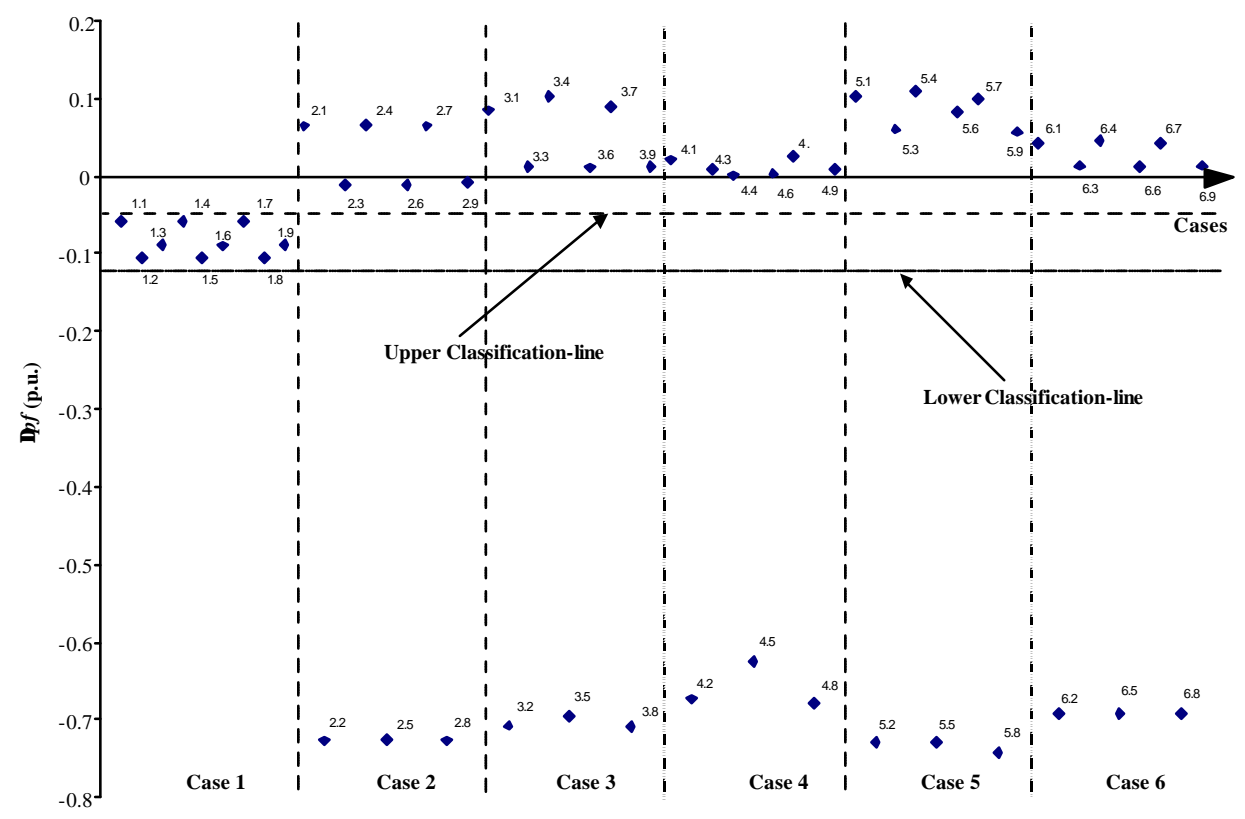

Figure 3 Plot of Maximum $\Delta p f$ Values.

those of cases 2 and 4 are negative. Whereas $\Delta p f$ can distinguish between case 1 (all negative) and cases 3-6, whose values are either positive or large negatives. Consequently a combination of $d V / d t$ and $\Delta p f$ can distinguish the LOM cases from all others. This can be best achieved by moving the classification-line shown in Fig 2 to the $x$-axis and therefore makes it easy to use $d V / d t$ for differentiating LOM, i.e., case 1 from all other cases except case 6 , i.e., LOPF. Similarly by shifting the
Upper classification-line (see in Fig 3 ) to the $x$-axis one can differentiate between LOM and cases 3-6 using $\Delta p f$. Figure 4 shows the resultant combined figure. Based on this figure it is possible to derive a suitable logic for a LOM relay as defined by the following equation:

$$
\text { Trip }=A \bullet B
$$

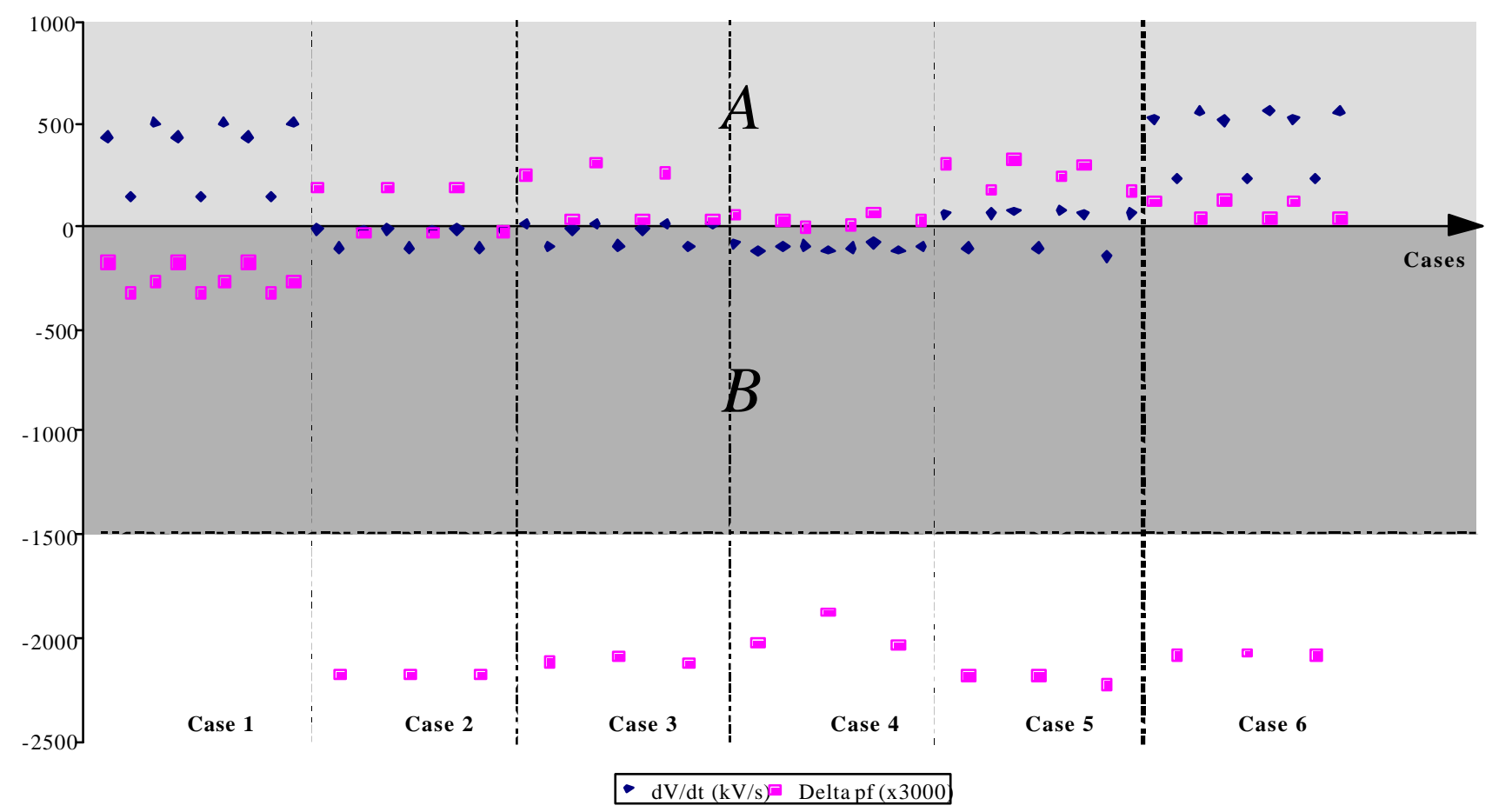

Figure 4 Combined Plots of $d V / d t$ and ${ }^{\Delta} p f$ for determining the values of $A$ and $B$. 
Where, $A$ and $B$ are defined by:

$$
\begin{aligned}
& A=\left\{\begin{array}{lc}
1, & \frac{d V}{d t} \geq 0 \\
0, & \text { otherwise }
\end{array}\right. \\
& B=\left\{\begin{array}{cc}
1, & 0 \geq \Delta p f \geq-0.5 \\
0, & \text { otherwise }
\end{array}\right.
\end{aligned}
$$

A and B are represented graphically using Fig 4, where it can be seen that the lighter shaded area is for all values greater than zero. Therefore, if the $d V / d t$ value is in this area then $A=1$, otherwise $A=0$. The darker shaded area coincides with $0 \geq \Delta p f \geq-0.5$. (Note that the $\Delta p f$ value has been multiplied by 3000 to make the diagram clearer, therefore $-0.5=-1500$ ). Thus $B=1$ if $\Delta p f$ falls within this area, else $B=0$. Therefore, for the trip output to equal 1 both $A$ and $B$ must also equal 1 . It should be clear from figure 4 that this can only happen for all case 1 results, i.e. for LOM occurrences.

Figure 5 illustrates how this could be implemented practically. The instantaneous voltage, $v(t)$, and current, $i(t)$, are sampled by a D/A converter. The sampled values are then used to calculate $d V / d t$ and $\Delta p f$. Suitable combinational logic is then used to determine the values of $A$ and $B$ and decides whether a trip signal is initiated or not.

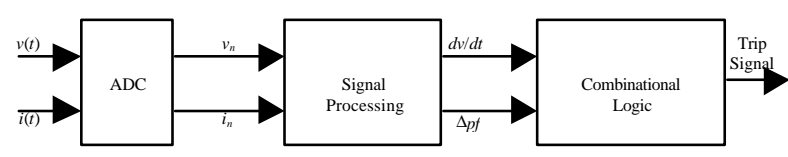

Figure 5 Diagram for LOM detector

\section{CONCLUSIONS}

This paper has reported an the development of a new algorithm for the detection of LOM for EGs. The new approach particularly deals with rather two difficult situations. These are (i) the case where the EG is "islanded" with a load of similar capacity to that of EG and (ii) the situation that requires differentiation between LOM and LOPF. The algorithm is based on continuously monitoring the rate of change voltage and the change of the power factor at the interfacing point between EG and the associated network. These can be derived from the instantaneous voltage and current signals measured at the interfacing point.

\section{ACKNOWLEDGEMENTS}

The authors would like to thank The Robert Gordon University for providing facilities, Ibrahim Rida for his invaluable experience with EMTP, Southern Electric for participating in discussions and providing site test, and ALSTOM T\&D and the EPSRC for their assistance and financial support.

\section{REFERENCES}

1. Salman S.K. and Mollah A.R., 1989, "Factors influencing the interconnection between private generators and public network,", UPEC'89, UK, 69-72

2. Redfern M.A., et al, 1997, "A new loss of grid protection based on power measurement", IEE Pub. No. $\underline{434}, 91-94$

3. Salman S.K. and King D.J., "Difficulties in using EMTP for investigation of loss of mains", Proceedings of the 33rd. Universities Power Engineering Conference, Edinburgh, UK, 8-10, September, 1998, Vol. 1, 17-20,

4. Salman S.K. and King D.J., 1999, "Monitoring changes in system variables due to islanding condition and those due to disturbances at the utilities, network", IEEE Trans \& Distrib Conf.. 2 , 460-465

5. Salman S.K. and King D.J. and Heggie G., 1999, "Modelling a ROCOF relay using EMTP”, UPEC' 99 2, 454-457

6. Warin J. and Allen W.H., 1990, "Loss of mains protection," ERA Conf. on Cir. Protec. for Ind. \& Comm. Inst, UK, 4.3.1-12

7. Cooper C.B., 1989, "Standby generation-problems and prospective gains from parallel running," Int. Conf. in Pow Sys. Protec, Singapore, 1-6

8. Rogers W.J.S., 1993, "Overview of embedded generators in public electricity networks," IEE Colloq., Chester, UK, 1/1-5

9. O'Kane, P and B Fox, 1997, "Loss of mains detection for embedded generation by system impedance monitoring", IEE Pub. No. 434, 95-98

10. Salman, S. K., 1997, "Detection of embedded generator islanding condition using elliptical trajectory technique", IEE Pub. No. 434, 103-106.

11. J. Russell, 1995, "Protection relays for mains failure with respect to G59," ERA Conf on Protec. Elec. Net. \& Qual. Sup. in a De-Regul. Ind., 7.3.1-10

12. King D.J., 1999, "New methods for the protection of embedded generators against the loss of utility networks," Ph.D. Thesis, The Robert Gordon University, 104-121 
